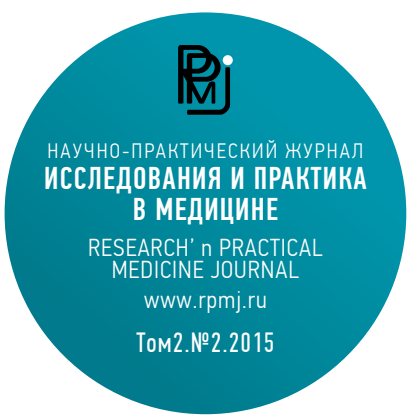

Ключевые слова: платинорефрактерный уротелиальный рак, винфлунин

Keywords: platinum-refractory urotelial cancer, vinflunine

DOI: 10.17709/2409-2231-2015-2-2-29-34

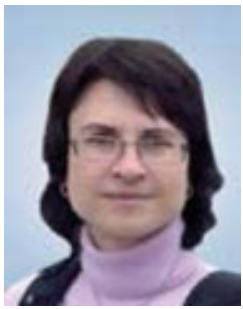

Для корреспонденции: Болотина Лариса Владимировна, д.м.н., заведующая отделением химиотерапии МНИОИ им. П.А. Герцена - филиал ФГБУ «НМИРЦ» Минздрава России

Адрес: 125284, Российская Федерация,

г. Москва, 2-ой Боткинский проезд, дом 3

E-mail: mnioi@mail.ru

Статья поступила 06.05.2015,

принята к печати 11.06.2015

For correspondence:

Bolotina Larisa Vladimirovna -

$\mathrm{PhD}, \mathrm{MD}$, head of the chemotherapy

Department of P. Hertsen MOR

2-y Botkinskiy proezd, 3, Moskva

125284, Russia

E-mail: mnioi@mail.ru

The article was received 06.05.2015,

accepted for publication 11.06 .2015

\section{СОВРЕМЕННЫЕ ПОДХОДЫ К ЛЕКАРСТВЕННОЙ ТЕРАПИИ РАКА МОЧЕВОГО ПУЗЫРЯ ПОСЛЕ ПЕРВОГО ПРОГРЕССИРОВАНИЯ}

\author{
Болотина Л.В., Королева Л.А.
}

МНИОИ им. П.А. Герцена - филиал ФГБУ «НМИРЦ» Минздрава России

125284, Российская Федерация, г. Москва, 2-ой Боткинский проезд, дом 3

\section{Резюме}

Количество цитостатиков, эффективных при раке уротелия, крайне мало. Базисным препаратом для режимов 1 линии остается цисплатин, применение которого, однако, может ограничиваться значимыми токсическими реакциями, которые развиваются у большого числа больных. В случае невозможности применения платиновых производных лечение может ограничиваться применением гемцитабина в режиме монотерапии. Значительно сложнее решать вопрос в отношении больных, прогрессирующих после 1 линии лечения. Эффективность ифосфамида, таксанов, топотекана, эпитилона В, пеметрекседа, nab-паклитаксела, пралатрексата, таргетных препаратов или их сочетаний не оправдали возлагаемых ожиданий.

Винфлунин (Жавлор) - инновационный химиотерапевтический препарат оказался эффективен для терапии платинорефрактерного уротелиального рака, в том числе в исследовании 3 фазы. Мы имеем собственный положительный опыт применения Жавлора у пациента старшей возрастной группы, представленный в статье.

\section{MODERN APPROACHES TO DRUG THERAPY OF BLADDER CANCER AFTER FIRST PROGRESSION}

Bolotina L. V., Koroleva L.A.

P. Hertsen MORI

2-y Botkinskiy proezd, 3, Moskva, 125284, Russia

\section{Abstract}

The number of drugs that is effective in urothelial cancer is very little. Cisplatin remains the basic drug for first line, but its use may be limited because of significant toxic reactions, that develop in a great number of patients. In case of impossibility of use of platinum derivatives treatment may be limited to the use of gemcitabine as monotherapy. It is much more difficult to resolve the issue regarding patients progressing after 1 st line treatment. The effectiveness of ifosfamide, taxanes, topotecan, epitilon B, pemetrexed, nabpaclitaxel, pralatrexate, targeted drugs or their combinations had not lived up to expectations.

Vinflunine (Javlor) is the innovative chemotherapeutic drug that is effective for the treatment of platinum-refractory urotelial cancer, including a phase- 3 trial. We have our own positive experience with Javlor in older age group of patients, that is presented in the article.

По данным зарубежной статистики выживаемость больных раком мочевого пузыря с восьмидесятых годов прошлого века находится на устойчивом плато. Количество цитостатиков, эффективных при раке уротелия, крайне мало. Попытки поиска новых активных противоопухолевых препаратов осложняются гетерогенностью опухоли и отсутствием определенных активирующих мутаций. Базисным препаратом для режимов 1 линии остается цисплатин, применение которого, однако, может ограничиваться значи- 
мыми токсическими реакциями, которые развиваются у большого числа больных. Таким образом, выбор терапии 1 линии основывается в первую очередь на характеристиках пациента, который может «подходить» или «не подходить» для наиболее эффективного, но и более токсичного варианта химиотерапии (XT). В случае невозможности применения платиновых производных, лечение может ограничиваться применением гемцитабина в режиме монотерапии. Значительно сложнее решать вопрос в отношении больных, прогрессирующих после 1-й линии лечения. Долгие годы для них существовал недостаток терапевтических опций после прогрессии заболевания и большинству предлагалась лишь симптоматическая или поддерживающая терапия.

С целью определения препарата, эффективного после химиотерапии 1-й линии, проводилось достаточно большое число исследований 2 фазы, однако по их результатам ни один из препаратов не был отобран для дальнейших исследований 3 фазы. Так, предпринимались попытки использования ифосфамида, таксанов, топотекана, эпитилона В, пеметрекседа, nab-паклитаксела, пралатрексата, которые не оправдали возлагаемых ожиданий. Несмотря на то, что при использовании некоторых цитостатиков непосредственный эффект составил около 30\%, медиана времени до прогрессирования (ВДП) не превысила 6 месяцев [1-4]. Так, например, назначение гемцитабина в трех небольших исследованиях сопровождалось общим ответом (полная ремиссия + частичная ремиссия, ПР+ЧР) равным 11\%-39\% с медианой общей продолжительности жизни от 5 до 13 месяцев. Однако большинство больных в настоящее время получают гемцитабин в 1-й линии лечения. Общий ответ от еженедельного применения паклитаксела также в небольших исследованиях колебался от 7\% до 9\% с медианой общей выживаемости (ОВ), несколько превысившей полгода (7 месяцев) [5]. Похожие результаты были представлены Cervera-Grau на конгрессе ASCO в 2012 для пеметрекседа [6]. Так, время до прогрессирования заболевания при назначении препарата во 2 линии терапии составило 4,2 месяца, а медиана общей продолжительности жизни вновь оказалась 7 месяцев.

Исследования по комбинированной XT также не дали четкого понимания выигрыша от той или иной лечебной комбинации, а исследования 3-ей фазы не подтвердили даже самые оптимистичные результаты, полученные во 2-й фазе. Так, комбинация паклитаксела и гемцитабина продемонстрировала непосредственную эффективность около $40 \%$, однако медиана времени до прогрессирования не превысила 4 месяцев, а медиана общей продолжительности жизни 8 месяцев [7]. Более того, не было проведено рандомизированных исследований III фазы, которые бы подтвердили эффективность этой комбинации во 2 линии

Также предпринимались попытки повторного использования платиносодержащих режимов во 2 линии лечения. В 2012 году Edeline с соавторами были опубликованы результаты исследования II фазы, оценившего возможность применения режима MVAC (метотрексат, винбластин, доксорубицин и цисплатин) у больных, получавших ранее комбинацию цисплатина и гемцита- бина (GC) [8]. На первый взгляд были получены очень оптимистичные результаты: общий ответ составил 61\%, время до прогрессирования болезни практически полгода (5,8 месяцев), а медиана общей продолжительности жизни превысила год и составила 14,2 месяца. Однако все эти результаты относились к общей популяции больных, включенных в исследование. При оценке результатов в подгруппах картина претерпела существенные изменения. Так, среди больных, которым режим GC применялся как адъювантная терапия после радикального хирургического вмешательства, общий ответ в рамках данного исследования составил $81 \%$ с медианой времени до прогрессирования 9,6 месяцев и 16,5 месяцев для ОВ. Но, по сути, у этой подгруппы больных режим MVAC мог рассматриваться как 1-ая линия лечения метастатического процесса. Более важными представляются результаты, полученные у больных с метастатической болезнью. Они оказались ожидаемо скромнее. В частности, на лечение ответили лишь $45 \%$ больных с медианой времени до прогрессирования 4,4 месяца и медианой продолжительности жизни менее полугода - 5,7 месяцев. Помимо этого исследуемый режим сопровождался существенной токсичностью: 3-4 степень отмечена на 69\% курсов, кроме того, зарегистрированы 4 случая летального исхода на фоне сепсиса, несмотря на применение колониестимулирующего фактора (КСФ) с 14-го дня лечения. В настоящее время принято считать возможным возвращение к режиму первой линии терапии, если время после ее завершения превысило 6-12 месяцев, у больного сохранена нормальная функция внутренних органов и пациент находится в хорошем общем состоянии.

Эффективность применения таргетных препаратов или их сочетания с цитостатиками в качестве 2 линии терапии вновь не позволили получить обнадеживающих результатов. Так, эффективность пазопаниба по данным разных авторов колебалась от $0 \%$ до $17 \%$ с медианой времени до прогрессирования от 1,4 до 3,3 месяцев. Воласертиб оказался эффективен у $14 \%$ больных с медианой времени до прогрессирования 1,4 месяца. Также малоэффективен оказался и эверолимус: непосредственный эффект был зарегистрирован лишь у 5\% больных, а медиана ВДП составила 3,3 месяца. Попытка блокады неоангиогенеза путем применения афлиберцепта тоже была неудачной - общий ответ удалось получить у 4,5\% больных, а медиана ВДП была менее 3 месяцев (2,8 месяца). Комбинация таргетных препаратов с цитостатиками оказалась малоэффективна - медиана ВДП составила 2,6 месяца с медианой общей продолжительности жизни лишь полгода [9]. Вот почему результаты терапии винфлунином пациентов прогрессирующим уротелиальным раком так привлекли внимание онкологов.

Винфлунин (Жавлор) - инновационный химиотерапевтический препарат для терапии платинорефрактерного уротелиального рака. Винфлунин является митотическим ядом, ингибирующим полимеризацию тубулина. За счет структурных изменений в катарантиновой части молекулы он отличается по эффективности от других винкаалкалоидов. Препарат оказывает два взаимодополняющих действия - цитотоксическое и ан- 
тиангиогенное. Важным является его обратимое взаимодействие с микротрубочками, за счет чего винфлунин обладает меньшей нейротоксичностью.

Оценивая клиническую эффективность винфлунина необходимо обратиться к двум открытым несравнительным клиническим исследованиям 2-й фазы, объединившим 200 больных, которым в качестве 2-й линии терапии назначалась монотерапия винфлунином [10, 11]. В рамках этих исследований была изучена непосредственная эффективность препарата (ОЭ), время до прогрессирования заболевания и общая продолжительность жизни больных. Необходимо отметить, что эти исследования включали в том числе достаточно тяжелую группу больных со сниженным клиренсом креатинина (40\%), наличием висцеральных метастазов (50\%) и коротким безрецидивным периодом после 1 линии терапии (менее полугода - до 70\%). Доза винфлунина составляла 280-320 мг/м², который вводился 1 раз в 3 недели. Полученные в обоих исследованиях результаты оказались многообещающими и воспроизводимыми. Так, ОЭ составила 15\%-17\%, контроля над болезнью удалось достичь у 60\%-65\% больных с медианой продолжительности жизни 7-8 месяцев. Для подтверждения полученных результатов было проведено исследование 3-ей фазы, в котором изучалась эффективность винфлунина в сравнении с наилучшей поддерживающей терапией (best supportive care, BSC) у больных, прогрессирующих после платиносодержащей XT [12]. Дизайн исследования очень прост. Триста семьдесят больных (из 83 онкологических центров) местно-распространенным или метастатическим уротелиальным раком, у которых наблюдалась прогрессия заболевания после 1-й линии лечения на основе платиновых режимов химиотерапии, делились в соотношении 2:1 в группы терапии винфлунином (n=253) в сочетании с BSC или в группу, получавшую лишь наилучшую поддерживающую терапию $(\mathrm{n}=117)$. Вин-

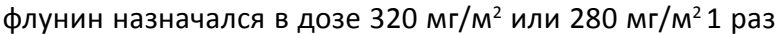
в 3 недели, лечение продолжалось до прогрессии заболевания. Основной целью исследования была оценка общей продолжительности жизни (ОВ). Исследование считалось успешным при разнице в ОВ между группами 2 и более месяцев. Кроме того, в работе предполагалось выявление возможных прогностических факторов (уровень щелочной фосфатазы (ЩФ), гемоглобина, наличие висцеральных метастазов, общее состояние больного, поражение лимфоузлов, проведение ранее лучевой терапии). Подавляющее число больных, включенных в исследование, были в хорошем общем состоянии, но 75\% пациентов имели висцеральные метастазы, а у $40 \%$ из них было поражено более 3 органов. Первичный анализ был проведен через 1,8 года наблюдения. По результатам проведенного анализа исследование оказалось успешным, основная цель была достигнута. Так, разница в медиане общей продолжительности жизни превысила 2 месяца между группами (6,9 мес. vs 4,3 мес., p=0,040), а снижение риска смерти от болезни снизилось на $23 \%$.

Среди факторов, влияющих на ОВ, были выявлены такие как: уровень щелочной фосфатазы (ЩФ), гемоглобина, наличие висцеральных метастазов, общее состояние больного и, конечно, проведение терапии винфлунином $(p=0,036)$. При проведении обновленного анализа было отмечено сохранение выигрыша в пользу применения винфлунина при наблюдении за больными более 3 лет. Так, к 30 месяцам наблюдения в группе терапии винфлунином оставались живы 14 больных в сравнении с 4 пациентами группы BSC, а 40 месяцев пережили 6 больных лечебной группы и никто из группы сравнения [13]. В проведенном исследовании были поставлены дополнительные (вторичные) цели по оценке непосредственной эффективности терапии (ОЭ), а также времени до прогрессирования заболевания (ВДП). По всем изученным показателям был отмечен выигрыш в пользу применения винфлунина. В частности, Оэ оказалась $8,6 \%$ vs $0 \%(p=0,0063)$, медиана ВДП 3,0 мес. vs 1,5 мес. ( $p=0,0012)$. Важным также является то, что контроля над болезнью (ОЭ + стабилизация) удалось достичь у 41,4\% больных лечебной группы в сравнении с 24,8\% пациентов группы сравнения ( $p=0,0024)$. Из токсических реакций 3-4 степени наиболее значимой оказалась нейтропения, которая развилась на половине курсов винфлунина, однако, фебрильная нейтропения была зарегистрирована лишь на 6\%. Из негематологических проявлений наблюдались астения (19,3\%), запоры $(16,1 \%)$ и тошнота $(2,4 \%)$, которые контролировались применением стандартных мер профилактики. Специфическими при использовании винфлунина оказались миалгии (3,2\%) и сенсорная нейропатия (1,2\%), которые не регистрировались в группе сравнения. Таким образом, можно констатировать, что токсические реакции на фоне терапии винфлунином являются предсказуемыми, имеют приемлемый профиль, легко управляются. Токсичность не является кумулятивной.

К началу 2013 года винфлунин зарегистрирован в большом числе стран Европы, Латинской Америки и России в качестве монотерапии пациентов распространенным или метастатическим уротелиальным раком при прогрессировании после платиносодержащих режимов.

Винфлунин назначается в 3-недельном режиме в рекомендуемой дозе $320 \mathrm{mr} / \mathrm{m}^{2}$ в виде 20-минутной инфузии. Обязательным является исследование показателей кроветворения перед назначением каждой дозы препарата. Необходимо особо подчеркнуть, что данная доза является стандартной для пациентов в хорошем общем состоянии (performance status, PS 0) и без предшествующей лучевой терапии на область таза. Дозировка должна быть снижена до $280 \mathrm{mr} / \mathrm{M}^{2}$ в случае предшествующей радиотерапии, а также для больных с PS 1. Если после 1 цикла лечения в указанных дозировках не было зарегистрировано гематологических или другого рода токсических реакций 3-4 степени, дальнейшее лечение должно быть продолжено в дозе 320 мг/м².

Существуют особые группы пациентов, которым уже на старте лечения необходимо проводить коррекцию дозы препарата. Так, у больных в возрасте 75-79 лет введение винфлунина должно проводиться в дозе $280 \mathrm{Mr} / \mathrm{M}^{2}$ даже в случае отсутствия в анамнезе облучения таза и при хорошем общем состоянии. Для пациентов же старше 80 лет доза винфлунина должна быть снижена до 250 мг/м² на курс. Помимо возраста для определения необходимой дозы препарата учитывается наличие 
у больного почечной и печеночной недостаточности. При умеренной почечной недостаточности (клиренс креатинина 40-60 мл/мин) используется доза $280 \mathrm{mr} / \mathrm{m}^{2}$, а при наличии тяжелой степени (клиренс креатинина 20$40 \mathrm{mл/мин)} \mathrm{доза} \mathrm{редуцируется} \mathrm{до} 250 \mathrm{mr} / \mathrm{m}^{2}$. Редукция дозы требуется и у больных с печеночной недостаточностью. Для пациентов с признаками печеночной недостаточности легкой степени (класс А по шкале Чайлд-Пью) начальная доза составляет $250 \mathrm{mr} / \mathrm{M}^{2}$ со снижением для больных с печеночной недостаточностью умеренной степени (класс В по шкале Чайлд-Пью) до 200 мг/.².

Резюмируя вышеизложенное, можно заключить, что винфлунин продемонстрировал похожую, клинически значимую эффективность в качестве терапии 2 линии уротелиального рака в исследования II и III фазы. Препарат имеет предсказуемый, приемлемый и управляемый профиль токсичности. Из токсических реакций наиболее значимыми являются нейтропения, астения и запоры, которые корригируются использованием стандартных средств профилактики. Важным результатом исследования III фазы является демонстрация положительного лечебного выигрыша в отношении общей выживаемости у больных, которым был назначен винфлунин в сравнении с пациентами, получавшими лишь поддерживающую терапию, который сохранялся при наблюдении за больными более 3-х лет. В рамках этого исследования удалось определить факторы риска прогрессирования у пациентов, предлеченных платиносодержащими режимами ХT.

Таким образом, на сегодняшний день Жавлор может быть признан новым стандартом лечения местно-распространенного или метастатического уротелиального рака при прогрессии после платиносодержащих режимов химиотерапии.

В качестве примера эффективного использования винфлунина приводим следующее клиническое наблюдение: больной С., 81 год. В 2011 году по поводу рака

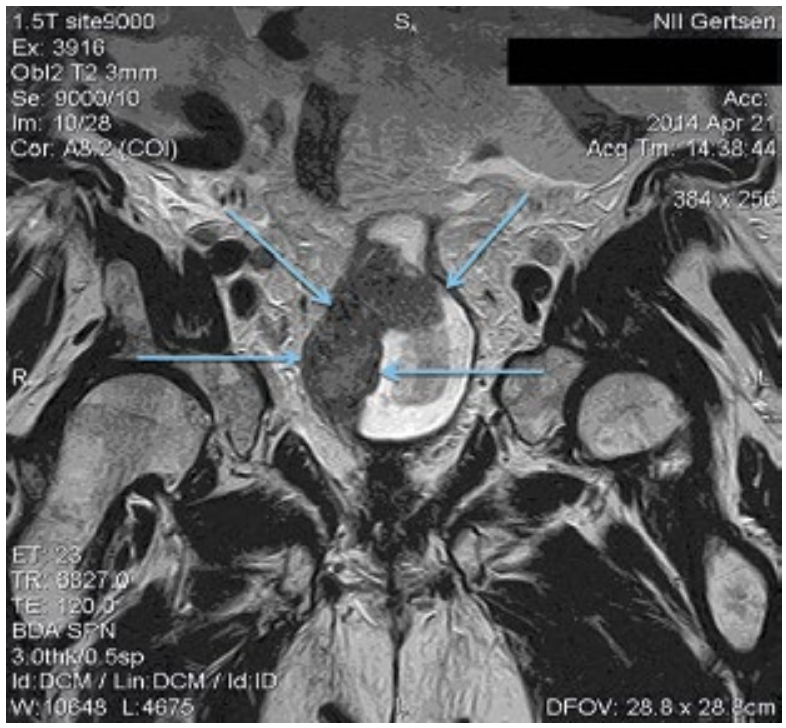

Рис.1. До начала лечения винфлунином мочевого пузыря I стадии перенес ТУР. Гистологическое заключение - переходно-клеточный рак, G2. Через 2 года, в марте 2013 года диагностировано прогрессирование заболевания в виде массивного рецидива в мочевом пузыре с вовлечением правого мочеточника, шейки мочевого пузыря, выходом в паравезикальную клетчатку. Гистологическое заключение - переходноклеточный рак G3. Обратился в отделение химиотерапии МнИОИ им. П.А. Герцена, где с апреля по сентябрь 2013 года проведено 6 курсов ПХТ 1-й линии гемцитабином и цисплатином с достижением частичной регрессии опухоли. В течение полугода пациент находился под динамическим наблюдением. В марте 2014 года зафиксировано прогрессирование заболевания, возобновление гематурии. С апреля 2014 года начато лечение винфлунином в дозе $280 \mathrm{mr} / \mathrm{M}^{2}$ (курсовая доза $550 \mathrm{Mr)} \mathrm{(рис.} \mathrm{1).}$ Уже после 1 курса терапии отмечено прекращение макрогематурии, нормализация показателей мочевины и креатинина. При контрольном обследовании после 2 курсов зарегистрирована положительная динамика в виде регрессии опухолевых очагов на 50,3\% (ЧР). После дополнительных 2 курсов лечения отмечено дальнейшее сокращение опухоли на $20 \%$. Таким образом, общий эффект составил 70,3\% (рис. 2). В целом пациенту проведены все запланированные 6 курсов терапии винфлунином. Из токсических реакций зафиксирована нейтропения 3 степени после 2 курса, введение КСФ не проводилось. Показатели гемопоэза самостоятельно восстановились на 5 день. Гематологическая и гастроинтестинальная токсичность сохранялись на протяжении всех последующих курсов, из которых преобладающими были тошнота, астения, анемия и нейтропения. Данное клиническое наблюдение позволяет заключить, что применение винфлунина в стандартных дозах у пациентов старше 80 лет не сопровождается выраженными токсическими реакциями, требующими массивной корригирующей терапии или отмены химиотерапии. Объектив-

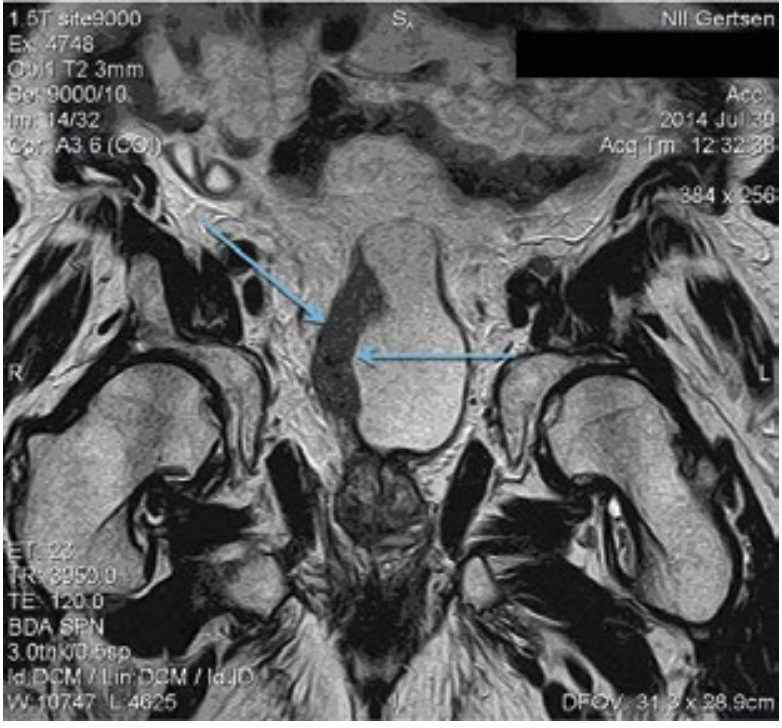

Рис. 2. После 4 курсов винфлунином 
ный ответ опухоли на лечение и приемлемый уровень токсичности подтверждают обоснованность назначения винфлунина пациентам уротелиальным раком старшей возрастной группы, получавших ранее платиносодержащую химиотерапию.

Помимо того, что подтверждена эффективность винфлунина в терапии 2-й линии уротелиального рака, в настоящее время предпринимаются попытки оценить возможность его использования в начале лечения метастатического процесса. Так, инициированы несколько исследований, в которых винфлунин-содержащие режимы применяются в качестве 1-й линии терапии. Одно из исследований - JASINT - объединяет 69 пациентов с противопоказаниями к применению цисплатина, которые разделены на 2 группы [14]. Одна группа получает комбинацию винфлунина с гемцитабином, вторая винфлунин с карбоплатином. Первичной целью исследования является оценка контроля над болезнью (ПР + ЧР + Стабилизация). В качестве вторичных целей выбраны оценка ОЭ, ВДП и ОВ. В 2014 году стали доступны результаты проведенного исследования. Оказалось, что уровень контроля над болезнью был равен в обеих группах: $76,5 \%$ vs 77,1\%. Также одинаковой была и медиана ВДП: 5,9 месяца vs 6,1 месяца. Медиана ОВ в цифровом выражении оказалась несколько больше в группе винфлунин + гемцитабин и составила 14,0 месяца в сравнении с 12,8 месяцев при замене гемцитабина на карбоплатин. Помимо этого, на фоне применения карбоплатина в качестве партнера для винфлунина существенно чаще развивались гематологические токсические реакции 3-4 степени. Так, нейтропения наблюдалась на 68\% курсов (фебрильная нейтропения 14\%), а тромбоцитопения на 21\% курсов. В сравнении с этим данные токсические реакции развились на $38 \%$ (фебрильная нейтропения $3 \%)$ и $6 \%$ курсов соответственно при использовании комбинации винфлунин + гемцитабин. Частота анемии ока-

\section{Список литературы/ References:}

1. Sweeney C.J., Roth B.J., Kabbinavar F.F., et al. Phase II study of pemetrexed for second-line treatment of transitional cell cancer of the urothelium. J Clin Oncol. 2006; 24(21): 3451-3457.

2. Vaughn D.J., Broome C.M., Hussain M., et al. Phase II trial of weekly paclitaxel in patients with previously treated advanced urothelial cancer. J Clin Oncol. 2002; 20 (4): 937-940.

3. Fechner G., Siener R., Reimann M., et al. Randomised phase II trial of gemcitabine and paclitaxel second-line chemotherapy in patients with transitional cell carcinoma (AUO Trial AB 20/99). Int J Clin Pract. 2006; 60(1): 27-31.

4. Kanai K., Kikuchi E., Ohigashi T., et al. Gemcitabine and paclitaxel chemotherapy for advanced urothelial carcinoma in patients who have received prior cisplatin-based chemotherapy. Int J Clin Oncol. 2008; 13 (6): 510-514.

5. Joly F., Houede N., Noal S., et al. Do patients with advanced urothelial carcinoma benefit from weekly paclitaxel chemotherapy? A GETUS phase II study. Clin Genitourinary Cancer. 2009; 7(2): E28-33;

6. Cervera Grau J. M., González-Billalabeitia E, Ayala F., et al. Long-term progression-free survival (PFS) and overall survival $(\mathrm{OS})$ to pemetrexed $(\mathrm{P})$ as single agent in metastatic urothelial carcinoma (MUC): A Spanish Oncology Genitourinary Group залась равной при применении обоих режимов (27\%). Негематологические токсические реакции были нечастыми (за исключением слабости - около 20\%) и наблюдались с одинаковой частотой в обеих группах. Таким образом, авторами сделаны два основных вывода о том, что оба изученных режима обладают равной эффективностью и позволяют обеспечить хорошие онкологические результаты у больных с наличием противопоказаний к применению цисплатина, однако комбинация винфлунина с гемцитабином обладает меньшей токсичностью и может быть адекватной альтернативой 1 линии терапии для больных распространенным уротелиальным раком в хорошем общем состоянии. Очень интересным представляется проводимое в настоящее время испанское исследование, которое ставит целью оценить эффективность поддерживающей терапии с переключением. Данная стратегия подтвердила свою эффективность на примере лечения рака легкого. В исследование MAJA включены 78 пациентов, которым по поводу распространенного процесса проведено 4-6 курсов индукционной терапии по схеме GC [15]. Далее половина больных, у которых не отмечено прогрессирования заболевания, оставлена на BSC, основной же группе к BSC добавлена поддерживающая терапия винфлунином в стандартном режиме. Основной целью исследования является оценка ВДП. К настоящему времени проведена лишь оценка безопасности, отмечен незначительный уровень токсических реакций, исследование продолжается.

Резюмируя вышеизложенное, можно констатировать, что винфлунин является единственным препаратом, продемонстрировавшим эффективность в отношении уротелиального рака, прогрессирующего после терапии 1 линии. Данный препарат может рассматриваться в перспективе адекватной заменой цисплатина в режимах 1-й линии после получения результатов проводимых в настоящее время исследований.

(SOGUG) systematic review. J Clin Oncol. 2012; 30(suppl; abstr 4587).

7. Suyama T., Ueda T., Fukasawa S., et al. Combination of gemcitabine and paclitaxel as second-line chemotherapy for advanced urothelial carcinoma. Jpn J Clin Oncol. 2009; 39(4): 244-250.

8. Edeline J., Loriot Y., Culine S., et al. Accelerated MVAC chemotherapy in patients with advanced bladder cancer previously treated with a platinum-gemcitabine regimen. Eur J Cancer. 2012; 48(8):1141-1146.

9. Choueiri T.K., Ross R.W., Jacobus S., et al. Double-blind, randomized trial of docetaxel plus vandetanib versus docetaxel plus placebo in platinum- pretreated metastatic urothelial cancer. J Clin Oncol. 2012; 30(5): 507-512.

10. Culine S., Theodore C., De Santis M., et al. A phase II study of vinflunine in bladder cancer patients progressing after firstline platinum-containing regimen. Br J Cancer. 2006; 94(10): 1395-1401.

11. Vaughn D.J., Srinivas S., Stadler W.M., et al. Vinflunine in platinum-pretreated patients with locally advanced or metastatic urothelial carcinoma: results of a large phase 2 study. Cancer. 2009; 115: 4110-4117. 
12. Bellmunt J., Theodore C., Demkov T., et al. Phase III Trial of vinflunine plus best supportive care compared with best supportive care alone after a platinum-containing regimen in patients with advanced transitional cell carcinoma of the urothelial tract. J Clin Oncol. 2009; 27(27): 4454-4461.

13. Bellmunt J., Fougeray R., Rosenberg J.E., et. al. Long-term survival results of a randomized phase III trial of vinflunine plus best supportive care versus best supportive care alone in advanced urothelial carcinoma patients after failure of platinum-based chemotherapy. Annals of Oncology. 2013; 24: 1466-1472.

Информация об авторах:

1. Болотина Лариса Владимировна, д.м.н., заведующая отделением химиотерапии МНИОИ им. П.А. Герцена - филиал ФГБУ «НМИРЦ» Минздрава России

2. Королева Людмила Алексеевна - к.м.н., врач отделения химиотерапии МНИОИ им. П.А. Герцена - филиал ФГБУ «НМИРЦ» Минздрава России
14. De Santis M., Wiechno P., Lucas C. et al. Mature survival data of a randomised international phase II trial (JASINT1): vinflunine (VFL) - gemcitabine (GEM) vs vfl- CBDCA in CDDP-unfit patients (PTS) with advanced urothelial carcinoma. Annals of Oncology. 2014; 25(Suppl 4): iv280-iv304.

15. Perez-Valderrama B., Villa J., Climent M., et al. Vinflunine maintance therapy vs best supportive care after platinum combination in advanced bladder cancer: a phase II, randomized, open label, study (MAJA), SOGUG 2011-12. Eur J Cancer. 2013; 49: 665.

Information about authors:

1. Bolotina L. V., PhD, MD, head of the chemotherapy Department of $P$. Hertsen MORI

2. Koroleva L.A., PhD, oncologist of the Department of chemotherapy of $P$. Hertsen MORI

\section{Оформление ссылки для цитирования статьи:}

Болотина Л.В., Королева Л.А. Современные подходы к лекарственной терапии рака мочевого пузыря после первого прогрессирования. Исследования и практика в медицине. 2015; 2(2): 29-34. DOI: 10.17709/2409-2231-2015-2-2-29-34

Bolotina L. V., Koroleva L.A. Modern approaches to drug therapy of bladder cancer after first progression. Issled. prakt. med. 2015; 2(2): 2934. DOI: 10.17709/2409-2231-2015-2-2-29-34

Конфликт интересов. Автор сообщает об отсутствии конфликта интересов. Conflict of interest. Author report no conflict of interest. 